Revue de l'Institut des langues et cultures

d'Europe, Amérique, Afrique, Asie et Australie

37 | 2019

Des genres en Méditerranée : pratiques, représentations et transfert

\title{
Des genres en Méditerranée : pratiques, représentations et transfert
}

Genres and Gender in the Mediterranean: Practices, Representations and

Circulation

Salam Diab-Duranton et Abdenbi Lachkar

\section{OpenEdition}

Journals

Édition électronique

URL : http://journals.openedition.org/ilcea/8292

DOI : 10.4000/ilcea.8292

ISSN : 2101-0609

Éditeur

UGA Éditions/Université Grenoble Alpes

Édition imprimée

ISBN : 978-2-37747-099-0

ISSN : 1639-6073

\section{Référence électronique}

Salam Diab-Duranton et Abdenbi Lachkar, « Des genres en Méditerranée : pratiques, représentations et transfert », ILCEA [En ligne], 37 | 2019, mis en ligne le 04 novembre 2019, consulté le 21 janvier 2020. URL : http://journals.openedition.org/ilcea/8292 ; DOI : 10.4000/ilcea.8292

Ce document a été généré automatiquement le 21 janvier 2020.

(C) ILCEA 


\title{
Des genres en Méditerranée : pratiques, représentations et transfert
}

\author{
Genres and Gender in the Mediterranean: Practices, Representations and \\ Circulation
}

Salam Diab-Duranton et Abdenbi Lachkar

1 La question du genre (littéraire, linguistique, médiatique, gender, etc.) a marqué l'histoire de l'homme depuis Aristote et continue d'être l'objet central de maintes recherches en sciences humaines et sociales comme en témoigne ce numéro 37 de la revue ILCEA. Les études qui y sont proposées entendent approfondir, parfois renouveler, tant les théories que les pratiques. C'est une manière de réinventer les sciences de l'Homme et de permettre aussi bien au chercheur qu'à son lecteur de s'interroger sur le pouvoir des genres à exprimer, écrire, décrire, représenter l'identité.

2 Au prisme des couleurs, des odeurs et des saveurs, vues, senties, appréciées, ici et ailleurs, tant en Méditerranée qu'en Orient, les présentes contributions - appartenant à plusieurs aires culturelles et venues de plusieurs universités au nord et au sud de la Méditerranée -, montrent combien la thématique du genre est fédératrice. Elle s'exprime au travers des manières d'écrire, de séduire, de plaire, de vivre en harmonie, voire d'enseigner. Écrire sur l'autre, étudier par exemple ses pratiques culinaires ou vestimentaires, est un acte de reconnaissance de la capacité universelle de l'homme à construire un dialogue entre les cultures. C'est une invite au rapprochement des individus, des groupes, des sociétés pour construire de nouvelles façons de penser et d'agir.

3 Irène Tamba (EHESS - Paris). Mare nostrum dans l'imaginaire collectif européen, la Méditerranée a longtemps pu être désignée par d'autres noms dans diverses langues, renvoyant à des couleurs vraies ou supposées de cet espace océanique. Irène Tamba reprend une question souvent traitée, discute les interprétations en cours, et démontre que ces dénominations peuvent renvoyer à trois usages distincts des couleurs des mers. 
Alternativement symbolique (dans des conceptions historiquement datées), physique (sous le regard de l'observateur) ou conventionnelle (dans la pratique cartographique).

Élisabeth Gavoille (université de Tours), à partir de L'Art d'aimer d'Ovide, analyse le lien entre séduction féminine et jeu des couleurs appliquées au visage. Celles-ci, (blanc, noir et rouge) par l'intensité de leurs contrastes expriment la vitalité de l'être et permettent de distinguer le féminin du masculin. La séduction féminine, naturelle ou artificielle, passe aussi par l'habit pour lequel Ovide prescrit des teintes douces et gaies, images $\mathrm{du}$ monde naturel (ciel et mer, fleurs et fruits). Ces teintes ou coloris, conjuguant le mythologique et le poétique, deviennent un symbole d'harmonie pour la femme. S'y réunissent les éléments essentiels pour se plaire à soi-même et se rendre séduisant à l'autre. L'artifice extrême s'y fait passer pour le parfait naturel.

Ana Maria Misdolea (Sorbonne Université), dans une perspective historique, fait appel aux notions de souveraineté, de purification et de fécondité pour traiter la thématique de la séduction. Elle étudie la «bonne odeur » dans la société romaine, comme élément contribuant à la mise en place d'une norme, puis comme moyen participant à la construction du genre féminin.

6 Ahyaf Sinno (université Saint-Joseph, Liban), inscrit sa recherche dans le cadre de la civilisation arabo-musulmane. Il étudie le zarf (raffinement) et les zurafă' (raffinés) dans le Muwaššă d'al-Waššā' (m. 325 / 937) qu'il s'agisse de textes poétiques ou de prose. Ce mouvement, que caractérisent subtilité, délicatesse et finesse, a regroupé des hommes et des femmes, et modelé les pratiques culturelles de plusieurs régions du monde arabo-musulman (Syrie, Iraq, Espagne musulmane, etc.) à diverses époques de leur histoire. Les "raffinés» ont fait de la notion d'Adab leur idéal, ce qui a eu des retombées directes sur leur comportement intellectuel et moral sans oublier leurs façons de s'habiller, de séduire, de se nourrir. Ahyaf Sinno évoque en ce sens les vêtements, la parure, les bijoux, les pierres précieuses et les parfums, mais aussi bien les aliments et les boissons dont les «raffinés » faisaient usage pour incarner leurs modes de vie. Il permet ainsi de mesurer l'impact qu'ils ont eu sur la culture méditerranéenne.

$7 \quad$ Nathalie Roelens (université du Luxembourg) traite de la notion de transfert culturel Est-Ouest, mais aussi Nord-Sud. Elle met en valeur des motifs ornementaux soumis à une forme de transhumance, migrant, comme les humains, d'un espace à un autre de la Méditerranée. Elle décrit le lien qui rattache l'homme (Ben Jelloun et Khatibi) à son environnement (Matera et Fès). Ce contexte vivant s'exprime en des couleurs vives et sublimes; il s'incarne dans des motifs visibles dans les formes, les corps et les genres. L'architecture, l'artisanat, l'art, aussi bien que la gastronomie en sont imprégnés. Pour sa démonstration l'auteure convoque à cette fin la synesthésie comme "puissance polysensorielle » transcendant les obstacles nés des nationalités et des confessions.

8 Mohammed El Fakkoussi (université Sidi Mohamed Ben Abdellah - Fès) fait appel à la notion de mésocosmos, empruntée à Ibrahima Sow, pour mettre au clair la question du merveilleux et du refoulement dans Une odeur de mantèque de Mohammed Khaïr-Eddine. Il y découvre une identité qui se vit dans l'imaginaire et par l'imaginaire, réservoir et miroir des productions psychosociales et des représentations socioculturelles des individus et des groupes vivant en société. L'auteur suggère que le déplacement ou le voyage, au lieu de constituer un enrichissement et de promettre un merveilleux, devient synonyme d'inculture, de déracinement, de refoulement dans la mise en scène 
de soi, de son corps, de ses propres odeurs, de celle du sein de sa mère Ainsi se constitue une identité inachevée et se construit une vision du monde mutilée.

Cléo Marmié (université Paul-Valéry Montpellier 3), conjuguant science politique, sociologie, esthétique et cultural studies, s'intéresse au street-art. Elle voit dans cette pratique artistique envahissant l'espace urbain marocain post-colonial le support de protestations ailleurs informulées. Pour elle, le street-art, oscillant entre le tolérable et l'intolérable, le légitime et l'illégitime, est une des manières utilisées par la jeunesse pour s'approprier l'espace public du Maroc actuel.

Elle met cette pratique en écho avec la situation socio-politique du monde arabe post " printemps arabe ", une société où tout est remis en cause (pouvoir, autorité, gestion politique). Par le biais de l'image, chemin faisant, elle étudie les différentes modalités d'expression, notamment dissensuelle, du street-art au Maroc. Elle suggère enfin les enjeux stratégiques de cette pratique artistique urbaine dans le cadre de la société marocaine et plus généralement méditerranéenne.

10 Souad Jadli \& Khadija Mouzon (université Hassan II - Casablanca) traitent du conte populaire comme genre littéraire, support et moyen de communication facilitant la conservation et la transmission d'un patrimoine oral très présent dans la culture méditerranéenne, notamment en France et au Maroc.

Les deux auteures s'intéressent en particulier au genre du conteur; elles constatent que cette pratique est toujours dominée par des conteurs hommes, le genre féminin, quant à lui, peinant à s'y faire une place. D'où leur proposition d'étudier les contes présents sur le web social (YouTube et Facebook) comme nouvel espace remplaçant les anciennes pratiques socio-culturelles.

11 Monica Masperi, Triscia Biagiotti, Giada Quaranta \& Martina Barletta (Université Grenoble Alpes) proposent une approche didactique sur les genres textuels avec l'objectif principal d'appréhender, dans le cadre du projet IDEFI Innovalangues, la diversité langagière et culturelle dans des formations de langues. Leurs réalisations didactiques se basent sur des séquences vidéo sélectionnées, principalement auprès des étudiants de niveau L1, contenant ou renvoyant à des valeurs culturelles plus ou moins représentatives de la langue-culture cible, notamment à l'oral. Les auteures, en plus de questionner les genres, s'interrogent aussi sur l'oral comme difficile objet d'enseignement-apprentissage sans la médiation de l'écrit.

12 Nabil Sukkari(université de Bourgogne Franche-Comté)

s'est proposé d'étudier, en se basant sur un corpus multimédiatique, l'impact de la crise

politique, pendant le « printemps arabe » et après, sur le discours touristique, perçu comme genre promotionnel en Égypte et en Tunisie. Il prend deux types de documents comme fils conducteurs : ceux de promotion et ceux de soutien. Ce qui lui permet d'observer, sous un angle sémiolinguistique et pragmatique, l'influence du contexte politique sur les techniques argumentatives utilisées. Félix Jun Ma (université Paul-Valéry Montpellier 3) s'intéresse à la découverte de la modernité, telle qu'observée dans le monde méditerranéen, qui s'exprime dans des écrits chinois à la fin de la dynastie des Qing (fin $\mathrm{XIX}^{\mathrm{e}}$ - début $\mathrm{Xx}^{\mathrm{e}}$ siècle). C'est à l'occasion de voyages personnels ou professionnels que les lettrés chinois cherchent des solutions à la crise que vit l'Empire du Milieu. Dans leurs récits de voyage, grâce à leur découverte de la Méditerranée et de l'Europe, ils développent une pensée progressiste. Ils rêvent d'une Chine modernisée qui allierait respect de la tradition et ouverture aux nouvelles idées. 

débat sur la relation Orients-Méditerranée à travers ce qu'il est convenu d'appeler les nouvelles routes de la soie. Ce projet considérable est le résultat direct de la puissance mondiale de la Chine, dont les projets politiques et socio-économiques influencent les pays du monde entier, excepté l'Amérique du Nord. L'auteur démontre que, en plus de l'axe ferroviaire Europe-Asie, l'axe portuaire liant la Chine, l'Asie du Sud-Est et du Sud, l'Afrique et l'Europe facilitera le commerce et augmentera les investissements chinois dans des secteurs stratégiques, notamment en Grèce. La Méditerranée est devenue le cœur de cet axe, la Grèce, sa tête de pont et la Chine son moteur financier.

Introduits par le rappel des noms servant à désigner la Méditerranée au long des siècles, les douze textes ici réunis couvrent une période temporelle longue, allant du $\mathrm{i}^{\mathrm{er}}$ siècle de notre ère à aujourd'hui. Ils concernent les pays méditerranéens, incluant Proche, Moyen et Extrême Orient. Y sont étudiés, à propos des genres, l'usage des couleurs dans la séduction féminine en Grèce ; la symbolique des odeurs, leur présence ou absence chez la femme romaine; le raffinement des hommes et des femmes dans la civilisation arabo-musulmane ; l'œcuménisme esthétique chez Matisse et Ben Jelloun ; la symbolique des odeurs chez Ibrahima Sow. Trouvent également leur place la dimension poétique et politique du street-art marocain comme nouvelle façon d'agir sur la société par l'art; le conte marocain comme tradition orale de transmission patrimoniale et sa valorisation par le web social, tout comme la représentation du monde méditerranéen dans les écrits chinois à la fin de la dynastie des Qing (fin XIX ${ }^{\mathrm{e}}$ début $\mathrm{xx}^{\mathrm{e}}$ siècle); les nouvelles routes de la soie, la Grèce constituant la tête de pont de la présence chinoise en Europe. Au total, les thématiques proposées dans ce numéro, par la diversité des questions traitées, la variété des approches théoriques, épistémologiques et didactiques, témoignent de la fécondité de l'approche genrée qui permet de renouveler des thèmes déjà souvent étudiés par ailleurs.

\section{AUTEURS}

\section{SALAM DIAB-DURANTON}

Université Grenoble Alpes

\section{ABDENBI LACHKAR}

Université Paul Valéry, Montpellier 